\title{
Haemagglutination inhibition technique for the demonstration of sulphones in urine *
}

\author{
H HUIKESHOVEN, MADELEINE DE WIT, \\ TA EGGELTE, JE LANDHEER \\ \& D L LEIKER \\ Royal Tropical Institute, Amsterdam, The Netherlands
}

Received for publication 24 October 1980

Summary A haemagglutination inhibition (HI) test for the detection of sulphones in urine is described. The lowest quantity of dapsone (DDS) in urine, detectable by $\mathrm{HI}$ is $1-0.1 \mu \mathrm{g} / \mathrm{ml}$. In urine samples collected from 10 volunteers sulphones are detectable by HI up to 3-6 days after taking single $100 \mathrm{mg}$ DDS doses. The method is less sensitive than the enzyme-linked immunosorbent assay (ELISA), described earlier, but its advantage is that only one incubation and no washing steps are required. This simple and specific test can be used to monitor self-medication of leprosy patients under field conditions.

\section{Introduction}

In a series of previous papers ${ }^{1-4}$, (unpublished data) we described an enzymelinked immunosorbent assay (ELISA) for the demonstration of sulphones in body fluids. This technique can be applied in 2 modifications to monitor leprosy-patient compliance, using either urine or blood specimens. The test is specific for sulphones, and the sensitivity is high. Since no sophisticated instruments are needed, the method can be used in leprosy endemic areas where laboratory equipment is often scarce.

Yet, due to its washing steps, the ELISA has not the simplicity of a spot test. However, such washing steps are absent in an haemagglutination inhibition (HI) technique, as described for the detection of drugs of abuse $\mathrm{e}^{5-8}$ and for the assay of gentamicin. ${ }^{9}$ A drawback of various haemagglutination techniques is

*This investigation received support from the Chemotherapy of Leprosy (THELEP) component of the UNDP/World Bank/WHO Special Programme for Research and Training in Tropical diseases, from the Netherlands Leprosy Relief Association (Nederlandse Stichting voor Leprabestrijding) and from the Italian Leprosy Relief Association (Amici dei Lebbrosi).

0305-7518/81/052229+07\$01.00 @ British Leprosy Relief Association 
the short shelf life of sheep red blood cells (SRBC), but fixation methods may overcome this problem. ${ }^{10^{-12}}$ Another limitation is the aspecific agglutination caused by substances present in normal urine specimens. However, when proper precautions are taken this aspecific reaction may be avoided. ${ }^{6,7}$

Because of its simplicity it was considered worthwhile trying to develop a $\mathrm{HI}$ in addition to the ELISA for sulphones. This paper describes a HI that detects sulphones in urine samples from volunteers who took single doses of $100 \mathrm{mg}$ dapsone (DDS).

\section{Materials and Methods}

\section{URINE SAMPLES}

Aliquots of urine samples collected earlier ${ }^{2}$ for testing the ELISA for sulphones were used. They were from 10 healthy volunteers who took single oral doses of $100 \mathrm{mg}$ DDS. The specimens were collected immediately before the DDS doses were taken and on 14 consecutive days thereafter.

\section{DDS-SENSITIZED SHEEP RED BLOOD CELLS (SRBC-DDS)}

Sheep blood was mixed $(1: 1)$ with modified Alsever's solution. ${ }^{10}$ The SRBC were separated by centrifugation $(10 \mathrm{~min}, 2000$ r.p.m. $)$ and washings $(5 x$ in saline, $2 x$ in PBS, $0.01 \mathrm{M}, \mathrm{pH}$ 7.2). Then they were suspended in a PBS volume equal to the cell volume.

Monodiazotized DDS was prepared by slow addition $\left(0^{\circ} \mathrm{C}\right)$ of an aqueous solution of $\mathrm{NaNO}_{2}(17.5 \mathrm{mg} / \mathrm{ml})$ to $124 \mathrm{mg}$ DDS dissolved in $5 \mathrm{ml} 1 \mathrm{~N} \mathrm{HCl}$, until slight turbidity occurred. The product was diluted 1:50 in PBS, and adjusted to $\mathrm{pH} 7.0$ using $1 \mathrm{~N} \mathrm{NaOH}$. One volume SRBC suspension was mixed (room temperature) with 10 volumes of the diazotized DDS solution. The product was shaken ( $2 \mathrm{~min}$ ), centrifugated (10 $\mathrm{min}, 2000$ r.p.m.), washed with PBS $(10 x)$ and saline $(3 x)$ and suspended in saline as an $8 \%$ suspension. This SRBC-DDS suspension was formalinized as described by Arquilla, ${ }^{10}$ and the stabilized cells were preserved at $4^{\circ} \mathrm{C}$ as a $50 \%$ stock suspension in PBS.

\section{HI DETECTION OF SULPHONES}

HI tests were performed in U-shaped wells of microtitre plates (Cooke), using $25 \mu \mathrm{l}$ aliquots of each of the following reactants:

(1) Supernatant of a test urine sample that was kept overnight at $4^{\circ} \mathrm{C}$, diluted $1: 10$ in PBS;

(2) Specific anti-sulphones Ig stock solution described previously, ${ }^{1}$ diluted 1:320 in PBS, or PBS without Ig for control tests, 


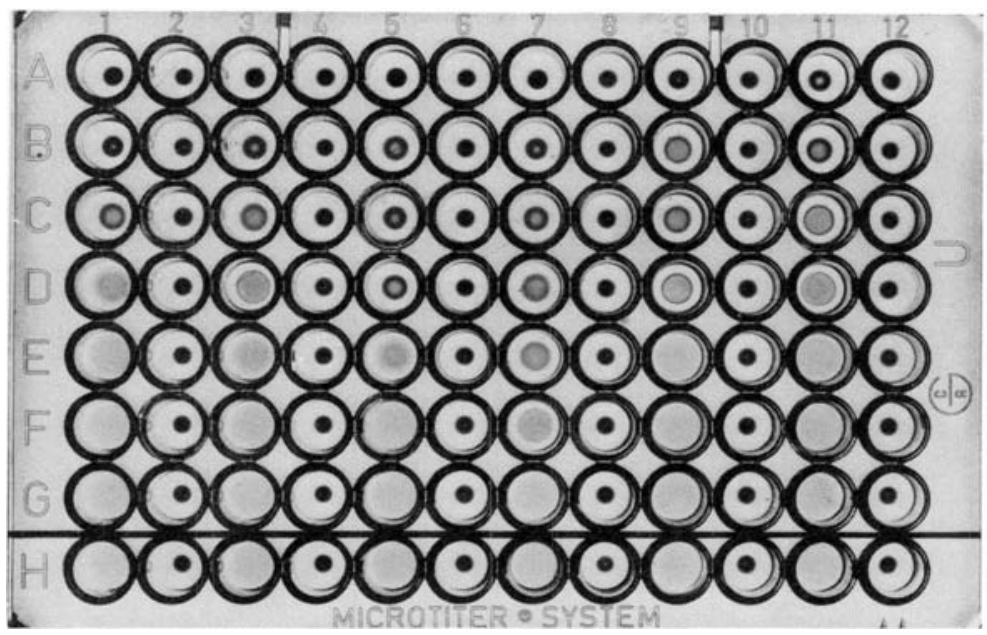

Figure 1. HI results on urine samples to detect sulphones. Row 1: mixed urine samples containing standard amounts of DDS, respectively 100 (A), 10 (B), 1 (C), 0.1 (D), 0.01 (E), $0.001(\mathrm{~F}), 0.0001(\mathrm{G})$ and $0(\mathrm{H}) \mu \mathrm{g} / \mathrm{ml}$. Rows 3, 5, 7, 9 and 11: urine samples from 5 different volunteers, collected respectively before treatment $(\mathrm{H})$, or on day 1 (A), 2 (B), 3 (C), 4 (D), 5 (E), 6 (F) and 7 (G) after taking $100 \mathrm{mg}$ DDS. Even rows are controls for aspecific $\mathrm{HA}$ of the samples in preceding odd rows.

\section{(3) SRBC-DDS stock suspension, diluted 1:15 in PBS.}

The $25 \mu 1$ aliquots were pipetted into the wells in this order. Urine and Ig were not preincubated as suggested by others, ${ }^{8}$ since in preliminary experiments this did not appear to influence the test. Results were read after 60 min incubation at room temperature. The optimal dilutions of the reactants were found by checkerboard titration.

\section{Results}

Figure 1 is a photograph of $\mathrm{HI}$ results using urine samples from 5 of the 10 volunteers. Even rows are controls for aspecific HA of the urine samples in preceding odd rows. In none of the 1:10 diluted specimens aspecific HA was detected, whereas in less diluted specimens this phenomenon frequently occurred. The first 2 rows of Figure 1 show results using standard amounts of DDS in urine. The lowest quantity of DDS detectable by HI in these standard samples is $1-0.1 \mu \mathrm{g} / \mathrm{ml}$ urine, i.e. $2.5-0.25 \mathrm{ng} /$ well (wells $\mathrm{C} 1-\mathrm{D} 1$ ). Also, in most urine samples collected up to 4 days after the $100 \mathrm{mg}$ dose of DDS sulphones could be detected by HI.

Since the same urine samples were analysed previously by the ELISA ${ }^{2}$ the results of both tests can be compared. Figure 2 is taken from the ELISA article. The odd rows show the same specimens as the corresponding rows in 


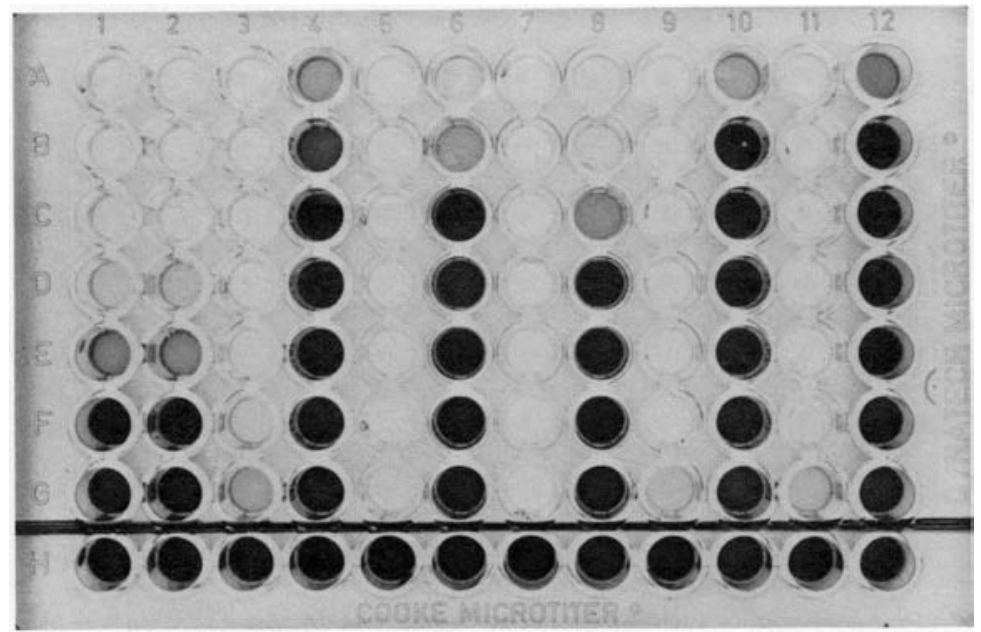

Figure 2. ELISA on urine samples to detect sulphones. Odd rows contain the same urine samples as those in Figure 1. Even rows are duplicate tests of samples in preceding odd rows (2 $\mathrm{A}-\mathrm{H}$, and $4,6,8,10,12 \mathrm{H}$ ), or samples collected on day 8 (A), 9 (B), 10 (C), 11 (D), 12 (E), 13 (F) and 14 (G) after taking $100 \mathrm{mg}$ DDS.

Figure 1. Row 2 is a duplicate of row 1, and the other even rows show ELISA results on days $8-14$ after the taking of $100 \mathrm{mg}$ DDS. Comparison of ELISA and HI results, using standard amounts of DDS in urine, indicates that HI is 10-100 times less sensitive than ELISA. Comparison of results on test samples illustrates that ELISA is positive for about 4 days longer than HI after single DDS dose. This would suggest a sixteen-fold difference in sensitivity, if calculations are based on an average $\mathrm{T} \frac{1}{2}$ (half-life) for DDS of about one day. ${ }^{2}$ The figures show that both in ELISA and HI, samples from the third volunteer (rows 7 and 8) were positive for longer than those from the others. A similar correspondence was seen in the samples obtained from a volunteer who was an extremely rapid DDS eliminator with an estimated T $\frac{1}{2}$ for DDS of $11 \mathrm{~h}$, as described in the ELISA paper. ${ }^{2}$ These samples (not shown in Figs 1 and 2) were negative sooner than average samples in both tests.

In Table 1 the numbers of urine samples positive by the two immunochemical methods are listed, together with the numbers positive by the quantitative DDS/creatinine (D/C) estimation, also reported in the ELISA paper. ${ }^{2}$ The table illustrates that in sensitivity the HI method takes an intermediate position between the ELISA and $\mathrm{D} / \mathrm{C}$ methods.

\section{Discussion}

The sensitivity of the HI test for sulphones is sufficiently high for monitoring leprosy-patient compliance with daily self-administration of $100 \mathrm{mg}$ DDS doses, 
Table 1. Comparison of ELISA, HI and D/C tests to detect sulphones in urine samples after single oral doses of $100 \mathrm{mg}$ DDS (10 subjects)

\begin{tabular}{rccc}
\hline $\begin{array}{c}\text { Time } \\
\text { (days) }\end{array}$ & $\begin{array}{c}\text { No. pos. } \\
\text { by ELISA }\end{array}$ & $\begin{array}{c}\text { No. pos. } \\
\text { by HI }\end{array}$ & $\begin{array}{c}\text { No. pos. } \\
\text { by D/C* }\end{array}$ \\
\hline 1 & 10 & 10 & 10 \\
2 & 10 & 10 & 10 \\
3 & 10 & 10 & 7 \\
4 & 10 & 9 & 3 \\
5 & 9 & 4 & 3 \\
6 & 9 & 1 & 1 \\
7 & 9 & 0 & 0 \\
8 & 9 & 0 & 0 \\
9 & 3 & 0 & 0 \\
10 & 1 & 0 & 0 \\
11 & 0 & 0 & 0 \\
12 & 0 & 0 & 0 \\
13 & 0 & 0 & 0 \\
14 & 0 & 0 & 0 \\
\hline
\end{tabular}

*Only $\mathrm{D} / \mathrm{C}$ ratios higher than the mean pretreatment value plus 3 standard deviations were considered positive.

although it lacks the potential of the more sensitive ELISA to distinguish between occasional and more substantial non-compliance. The difference in sensitivity between the 2 tests may be explained partly by the $1: 10$ dilution of urine samples to be tested in HI. This dilution is essential, for even in 1:8 diluted samples aspecific HA occurred. It is therefore advisable to test control aliquots of each urine sample for this phenomenon. For the same reason it is important to keep the test samples at $4^{\circ} \mathrm{C}$ for several hours, or preferably overnight, and use the clear supernatant. For the analysis of freshly voided specimens, centrifugation will be needed in order to obtain a supernatant.

The specificity for sulphones is high, since in preliminary experiments no cross-reaction with important sulphone analogues, the sulphonamides, was found. It shares this specificity with the ELISA, ${ }^{1,3}$ The strength of the HI test lies in its rapidity, simplicity and economy. Its advantage over the ELISA is the absence of washing steps, since no separation of reactants is required. Once the materials for the $\mathrm{HI}$ test are available it is as simple as a spot test.

Formalinized red blood cells, stored as long as 1 year at $4^{\circ} \mathrm{C}$, are known to show no significant alteration in their properties. ${ }^{10}$ It remains to be investigated whether the conjugation of DDS prior to the fixation, has any influence on this stability. In other HA assays the antigen was conjugated to cells that already were formalinized, ${ }^{10,12}$ but so far this procedure did not give satisfactory results with DDS. Suspensions of the red cell conjugates for other HA assays are reported to remain stable for many weeks. Such preparations can be used in HA after 1 washing with the diluent. When lyophilized after dilution to the final desired concentration, the sensitized cells can be used directly after 
resuspension in distilled water. ${ }^{10}$ The stability of the SRBC-DDS is currently being examined. Preliminary results both with resuspensions and lyophilized portions are comparable to those described. We are especially interested in the influence of tropical temperatures, which occur in most leprosy endemic areas. So far it could be shown that both suspensions and lyophilized portions of SRBC-DDS remained in optimal condition when kept at $37^{\circ} \mathrm{C}$ for 3 days. The stability of the specific Ig is quite satisfactory, as described earlier. ${ }^{3}$

We also examined the influence of elevated temperatures on the test itself. Identical results could be obtained in $\mathrm{HI}$ at room temperature, at $37^{\circ} \mathrm{C}$ and at $56^{\circ} \mathrm{C}$. However, an elevation of temperature required the use of a higher concentration of specific anti-sulphones Ig. The optimal incubation time of 60 minutes was similar at varying temperatures.

Based on these and further experiments the test will be adapted to field conditions in leprosy-endemic areas. It is hoped that the combination of immunochemical specificity with spot test simplicity will make the $\mathrm{HI}$ a valuable method to monitor DDS self-medication where required.

\section{Acknowledgements}

The authors are indebted to the volunteers for taking doses of DDS and giving the materials for this study.

\section{References}

1 Huikeshoven H, De Wit M, Soeters A, Eggelte TA, Landheer JE, Leiker DL. ELISA inhibition technique for the demonstration of sulphones in body fluids. I. Sulphones specific antibody-enzyme conjugate. Lepr Rev, 1979, 50, 275-81.

2 Huikeshoven H, De Wit M, Soeters A, Landheer JE, Leiker DL. ELISA inhibition technique for the demonstration of sulphones in body fluids. II. A new method to monitor leprosy patient compliance under field conditions. Lepr Rev, 1981, 52, 11-18.

${ }^{3}$ De Wit M, Huikeshoven H, Soeters A, Eggelte TA, Landheer JE, Leiker DL. ELISA inhibition technique for the demonstration of sulphones in body fluids. Comparison of two ELISA methods. Lepr Rev, 1981, 52, 125-130.

${ }^{4}$ Huikeshoven H, De Wit M, Soeters A, Landheer JE, Leiker DL, Niemer AQHJ, Warndorff T. ELISA inhibition technique for the demonstration of sulphones in body fluids. The use of dried blood on filter paper to monitor leprosy patient compliance. Lepr Rev, 1981, 52, 131-138.

5 Adler FL, Liu C-T. Detection of morphine by hemagglutination-inhibition. J Immunol, $1971,106,1684-5$.

${ }^{6}$ Adler FL, Liu C-T, Catlin DH. Immunological studies on heroin addiction. I. Methódology and application of a hemagglutination inhibition test for detection of morphine. Clin Immunol Immunopathol, 1972, 1, 53-68.

7 Liu C-T, Adler FL. Immunologic studies on drug addiction. I. Antibodies reactive with methadone and their use for detection of the drug. J Immunol, 1973, 111, 472-7. 
8 Decato L, Liu C-T, Adler FL. Immunological studies on drug addiction. III. Antibodies reactive with cocaine metabolites and their use for drug detection. J Immunol Methods, 1977, 18, 201-13.

9 Mahon WA, Feldman RI, Scherr GH. Hemagglutination inhibition assay for gentamicin. Antimicrob Agents Chemother, 1977, 11, 359-61.

10 Arquilla ER. Antigen-red cell conjugates prepared with bisdiazobenzidine. In: Williams CA, Chase MW, eds. Methods in Immunology and Immunochemistry. Volume IV: Agglutination, complement, neutralization and inhibition. New York, San Francisco and London: Academic Press, 1977, 41-7.

11 Mahan DE, Copeland RL. A method using toluene-2,4-diisocyanate and glutaraldehyde to stabilize and conjugate antigens to erythrocytes for use in passive hemagglutination tests. J Immunol Methods, 1978, 19, 217-25.

12 Assimeh SN, Johnson PM. A haemagglutination method for detection of rheumatoid factor using preserved erythrocytes covalently coated with human IgG. J Immunol Methods, 1980, 34, 205-15. 